\title{
Modélisation des agitations dans le Bassin d'Arcachon
}

\author{
Jean-Paul PARISOT ${ }^{1}$, Jeanne DIET-DAVANCENS ${ }^{1}$, \\ Aldo SOTTOLICHIO ${ }^{1}$, Elise CROSLAND ${ }^{1}$, Chloé DRILLON ${ }^{1}$ \\ et Romaric VERNEY ${ }^{2}$ \\ ${ }^{1}$ Equipe METHYS. UMR 5805-EPOC/OASU, Université Bordeaux 1. Avenue \\ des Facultés, 33405, Talence, \\ parisot@epoc.u-bordeaux1.fr \\ ${ }^{2}$ IFREMER Dyneco/PhySed BP 7029280 Plouzané \\ Romaric.Verney@ifremer.fr
}

\section{Résumé :}

Dans les zones intertidales soumises à de faibles conditions énergétiques, le clapot généré par le vent peut jouer un rôle prépondérant dans la remise en suspension des sédiments. L'objectif de ce travail est de tenter une première caractérisation du clapot dans le Bassin d'Arcachon, dans le but final de quantifier son impact sur la dynamique sédimentaire des estrans vaseux. Une campagne de mesures de hauteur et périodes des vagues a été réalisée de décembre 2006 à février 2007. L'analyse des données a permis d'observer un clapot de plus de $10 \mathrm{~cm}$ de haut lorsqu'un vent d'Ouest souffle à plus de $8 \mathrm{~m} / \mathrm{s}$, pouvant atteindre $40 \mathrm{~cm}$ pour des épisodes très venteux. Le code numérique SWAN a été appliqué afin de simuler le clapot, qui semble en bon accord avec les mesures.

\begin{abstract}
:
On intertidal zones subject to weak energy conditions, wind waves can play a leading role in the sediment dynamics. The aim of this study was to characterize wind waves in the Arcachon lagoon so that it was possible to quantify its impact on the sedimentary dynamics of the mud plates. To do that, a network of observation stations was set from December 2006 to February 2007, measuring at high frequency water height. The analysis of the hydrodynamic data enabled us to observe wind-waves with significant heights exceeding $10 \mathrm{~cm}$, when a west wind was blowing with velocities over $8 \mathrm{~m} / \mathrm{s}$ (40 cm with strong wind). The SWAN numerical model used in this paper gives a good prediction for the features of the wave generated by wind.
\end{abstract}

\section{Mots-clés :}

Clapot, hauteur significative, fetch, ALTUS, SWAN, Bassin d'Arcachon 


\section{$1 \quad$ Introduction}

Dans les vasières intertidales, les baies calmes et les systèmes lagunaires côtiers protégés par des barrières naturelles, la prise en compte des vagues sur la dynamique sédimentaire a fait l'objet d'un nombre croissant d'études récentes (Le Hir et al., 2000 ; Janssen-Stelder, 2000 ; Christie et al., 2001; Lee et al., 2004 ; Verney et al., 2006 ; Quaresma et al., 2007). En effet, ces environnements sont le plus souvent le siège d'une faible dynamique de courants de marée. C'est par exemple le cas de la lagune de Venise dans laquelle le vent est capable d'initier des processus contrôlant, à court terme ou durablement, la morphodynamique et la biorépartition (Rolinski et al. 2005). Ces sites sont généralement en accrétion sédimentaire puisque soumis à de faibles conditions énergétiques : la mer de Wadden dont la partie interne se comble (Pedersen et al., 2006) ou encore de la baie du Mont Saint Michel (Ehrhold et al., 2003) en sont des illustrations. Lorsqu'ils sont, de plus, protégés de la houle océanique, seul le vent est capable de rendre ces milieux plus énergétiques qu'ils ne le sont dans des conditions normales (Perillo et al. 1995). Le clapot correspond à l'état désordonné de l'agitation de surface engendrée localement par le vent. Dépendante du fetch (longueur du plan d'eau) et des caractéristiques éoliennes (l'intensité, la durée d'action et la direction conditionnant la longueur du fetch), l'agitation peut être irrégulière en direction, hauteur et période (comprise entre une et cinq secondes).

Le but de ce travail est de caractériser le clapot au sein d'une lagune côtière mésotidale atlantique, le bassin d'Arcachon. En effet, sur cette lagune il importe de connaître les forçages susceptibles de générer de la remise en suspension des sédiments fins, parmi lesquelles l'agitation due au vent. Cette étude repose a) sur l'interprétation d'une série d'enregistrements in situ obtenus récemment, b) sur l'application d'un modèle de propagation des vagues (SWAN, Booij et al., 2004) dont nous avons évalué la capacité à reproduire les ordres de grandeurs mesurées.

\section{$2 \quad$ Le Bassin d'Arcachon}

Le Bassin (Figure 1) est relié à l'océan Atlantique par un système de passes qui permet la propagation de l'onde de marée à l'intérieur de la lagune (Bouchet, 1968). La marée est de type semi diurne et d'amplitude mésotidale (marnage moyen compris entre 2 et 5 mètres). Il est établi que la marée et les courants qui lui sont associés jouent un rôle important sur les processus morphosédimentaires initiés à l'intérieur du bassin (Gayet, 1986), notamment la distorsion provoquée par le déphasage des ondes principales M2 et M4 (Salles et al. 2008). Les passes du Bassin (orientées selon un axe NE-SW) limitent la pénétration de la houle 
observée sur la côte Aquitaine (orientée souvent NW). Par diffraction, l'embouchure et la partie externe du Bassin sont partiellement affectées (Fenies, 1986). Un travail spécifique de modélisation de la houle sur les passes est effectué actuellement par Lecacheux et al. (2008). En revanche, la partie interne est bien protégée, et on peut considérer que la houle n'est pas un forçage significatif dans se secteur, raison pour laquelle ce travail se limite au clapot.

Le clapot est directement lié aux caractéristiques du vent. Les statistiques issues d'enregistrements faits au Sémaphore de la pointe du Cap Ferret montrent, qu'à l'échelle de l'année, les vents de Nord-Est sont à peine plus fréquents $(18,6 \%)$ que ceux d'Ouest $(15,6 \%)$ et de Nord-Ouest (15,1\%), (SOGREAH, 2000). En ce qui concerne le clapot résultant, la seule référence disponible à notre connaissance est celle de Gassiat (1989), qui a estimé visuellement des vagues pouvant atteindre $70 \mathrm{~cm}$ de hauteur, mettant en jeu une énergie considérable susceptible d'engendrer une remise en suspension des sédiments du fond. Dans ce contexte particulier (zone abritée de faible profondeur), l'agitation engendrée par le vent se propage facilement sur les estrans particulièrement larges de la zone interne du Bassin et affecte probablement la dynamique sédimentaire locale.

\section{Caractérisation expérimentale du clapot}

\subsection{Les mesures de vagues}

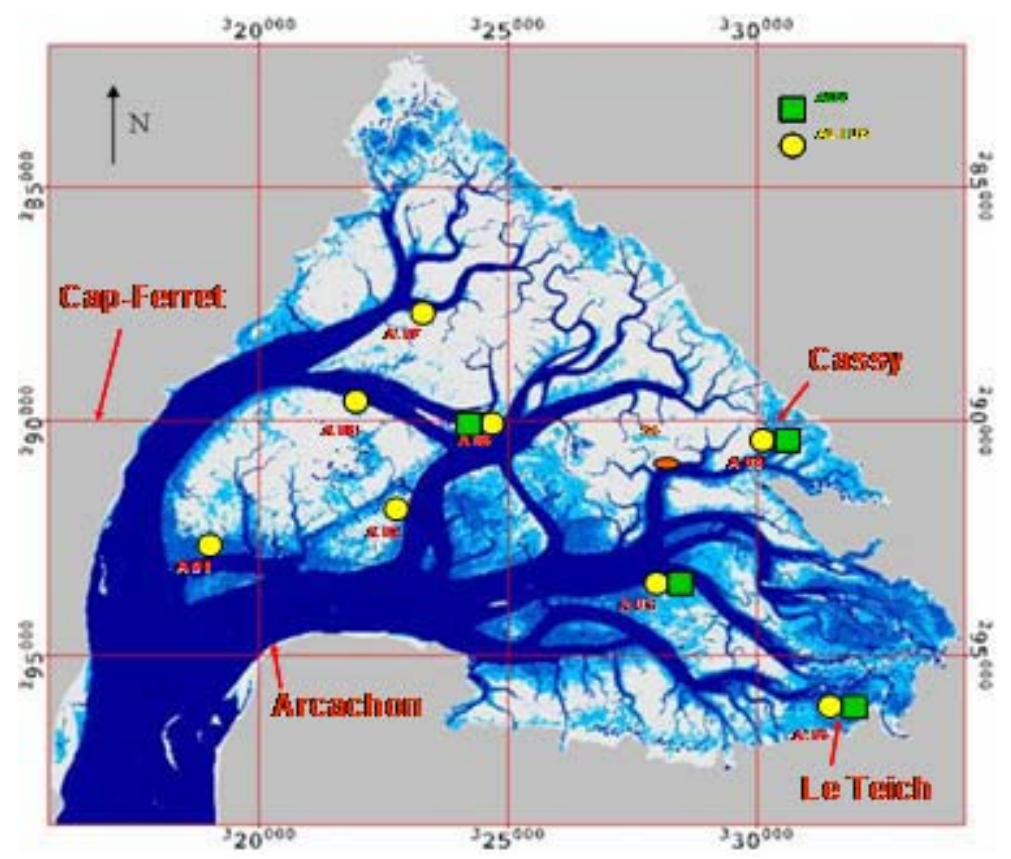

Figure 1. Localisation spatiale du dispositif expérimental. Les zones claires correspondent aux zones intertidales, les zones foncées caractérisent les chenaux. 
L'étude in situ, s'est déroulée du 4 décembre 2006 au 16 février 2007, dans le cadre du projet PNEC Chantier Littoral Atlantique. Huit points répartis sur toute la surface du bassin ont été instrumentés (figure 1). Les stations ont été positionnées sur des fonds vaseux ou sablo-vaseux, et toujours en zone intertidale, mais suffisamment près des chenaux pour limiter les temps d'exondation. Les stations numérotées de $\mathrm{A} 01$ à $\mathrm{A} 08$ ont été équipées avec des altimètres Altus. Quatre stations de la partie interne du bassin ont été équipées de vélocimètres ADV (non analysés ici). Les ALTUS dont les enregistrements font l'objet de ce travail (figure 2) sont des altimètres acoustiques enregistreurs submersibles, de haute précision (résolution $0,2 \mathrm{~mm}$, précision $\pm 5 \mathrm{~mm}$ ). Ce sont des systèmes autonomes permettant d'enregistrer les variations du niveau sédimentaire afin de quantifier un dépôt ou une érosion (Verney, 2006). Le principe de fonctionnement de l'Altus est basé sur l'émission et la réception d'une onde acoustique (1 MHz). L'instrument détermine à la fois la pression, donc la hauteur d'eau, ainsi que la distance entre les capteurs et le fond ce qui donne une indication sur l'évolution $\mathrm{du}$ fond (érosion ou engraissement). Les données étaient recueillies toutes les semaines. Une mesure est effectuée 2 fois par seconde, par burst de 302 points, soit 2 min 30 d'enregistrement toutes les 20 minutes. Ces instruments de mesures nous ont permis d'extraire la hauteur d'eau, la période et la hauteur significative des agitations. La hauteur significative est calculée à partir de l'écart-type des variations de hauteurs d'eau $\left(\mathrm{H}_{\mathrm{s}}=4 \sigma\right)$.

En complément du dispositif instrumental, nous disposons des données météorologiques périodiques de la station du Sémaphore, située sur la pointe du Cap Ferret. Les mesures de vent y sont effectuées par un anémomètre placé en hauteur, ramenées à un vent conventionnel soufflant à $10 \mathrm{~m}$ au dessus du sol.
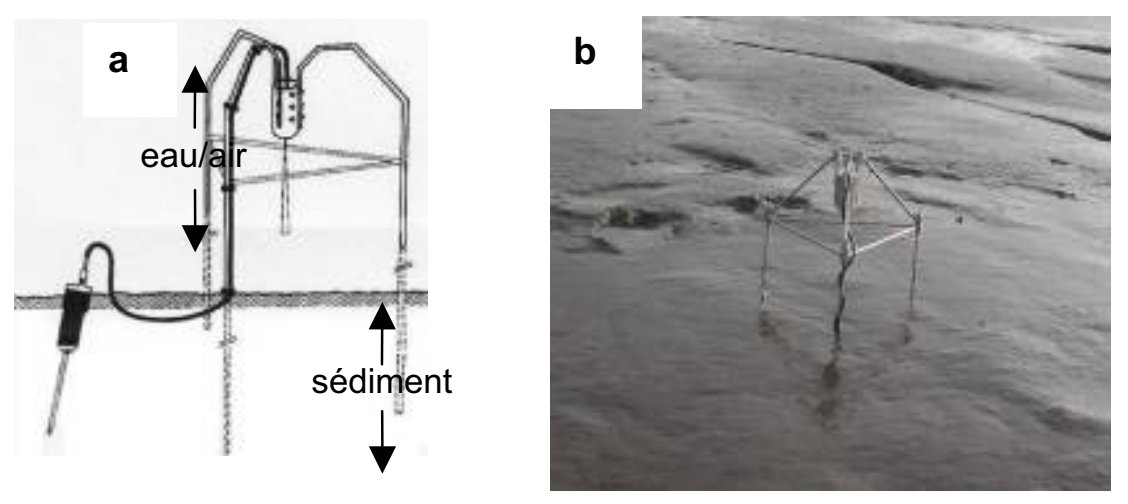

Figure 2. a) Schéma du système ALTUS ; b) Photographie de l'ALTUS A08 de la station atelier de Cassy 


\subsection{Les conditions de vent}

Nous disposons des mesures de vents tri-horaires (moyennes sur 10 minutes). Durant la campagne de mesures (figure 3), les vents enregistrés étaient principalement de Nord-Est $(35,8 \%)$, avec aussi une forte proportion de vents d'Ouest $(24,5 \%)$. Ces vents dominants caractérisent plus de $60 \%$ des vents répertoriés. On peut noter 4 événements très venteux les 8 décembre, 3 janvier, 18 janvier et 9 février.

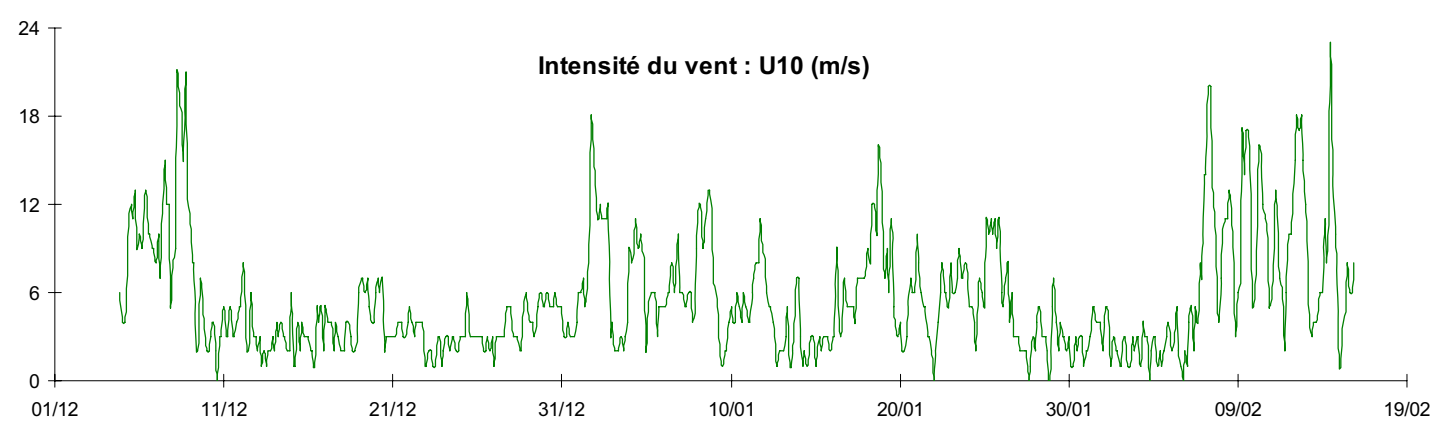

Figure 3. Intensité du vent durant la période de mesures Altus (décembre 2006 à février 2007)

La figure 4 intègre l'ensemble des hauteurs significatives de clapot enregistrées sur chacun des sites ; elle montre une agitation de surface permanente constituant une sorte de bruit de fond (d'une amplitude inférieure à $5 \mathrm{~cm}$ ), et la présence événementielle d'élévations plus ou moins fortes et plus ou moins étalées dans le temps. On peut noter la forte corrélation entre l'intensité du vent et la formation du clapot : dès que le vent dépasse $10 \mathrm{~m} / \mathrm{s}$, un clapot de plus de $10 \mathrm{~cm}$ se forme. Durant les événements très venteux, la hauteur caractéristique dépasse $20 \mathrm{~cm}$ pour atteindre près de $50 \mathrm{~cm}$.

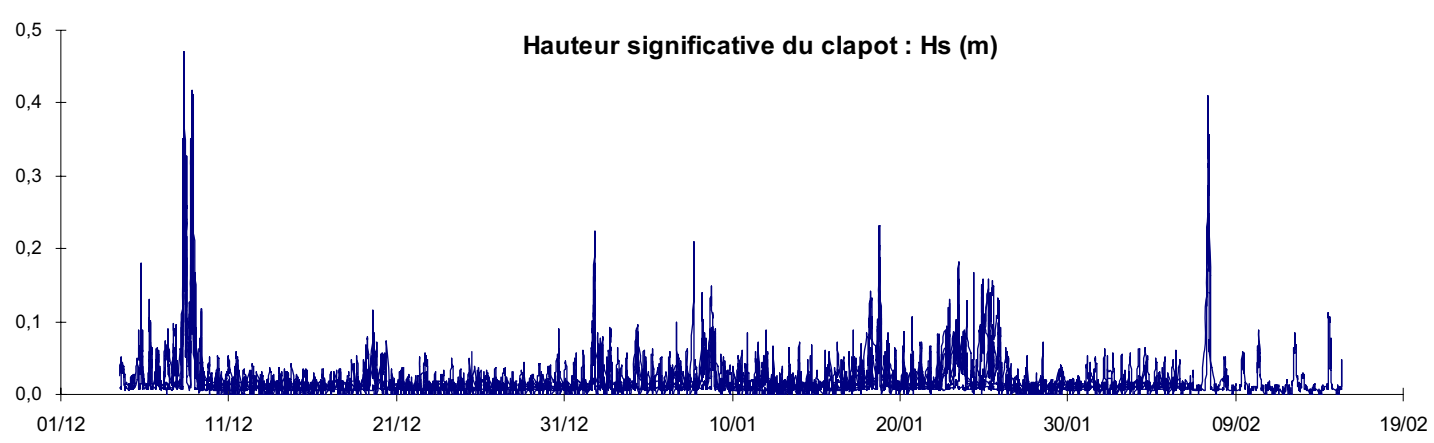

Figure 4. Hauteurs significatives du clapot. La chute brutale du signal le 9 février, est liée à un arrêt de l'instrument, dû à un défaut d'alimentation électrique. 


\subsection{Episode du 8 décembre}

La figure 5 permet de suivre en détail l'évolution des caractéristiques du clapot pendant deux marées consécutives, en deux stations à l'Ouest de la lagune (A04 et A08) : hauteur significative du clapot en noir (échelle de gauche en mètres), période du clapot en gris (échelle de droite en secondes), et hauteur du plan d'eau en bleu (échelle de droite en mètres) sur 3 sites. Les valeurs sont des moyennes par burst de $10 \mathrm{mn}$ toutes les $30 \mathrm{mn}$.

La hauteur significative augmente avec la hauteur d'eau. Toutefois, cette augmentation n'est pas linéaire. Tant que la hauteur d'eau ne dépasse pas un certain seuil (autour de 1,5 m), la hauteur significative n'augmente que très peu. Une fois ce seuil atteint, la hauteur significative augmente de manière exponentielle. Rapidement l'augmentation de la hauteur d'eau (au delà de 3,60 m) n'engendre plus d'augmentation de la hauteur significative. A ce stade là, la profondeur n'est plus un facteur limitant.
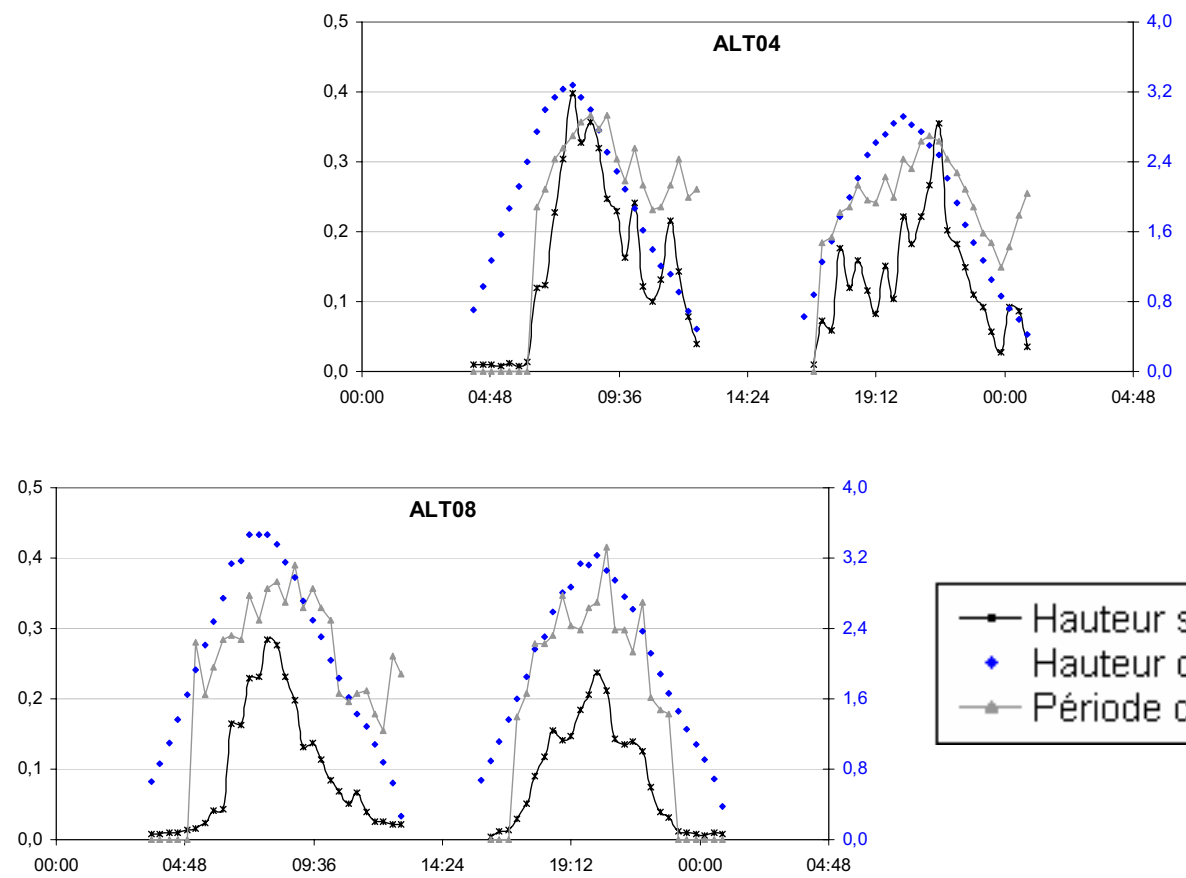

$\rightarrow$ Hauteur significative (m)

- Hauteur du plan d'eau (m)

$\rightarrow$ Période de l'onde (s)

Figure 5. Hauteur significative (échelle de gauche en mètres) et période (échelle de droite en secondes) du clapot, couplées à la hauteur du plan d'eau (échelle de droite en mètres), sur la période du 8/12/06 00h00 au 9/12/06 4h48, pour 2 des 8 sites ateliers du Bassin 


\section{Simulation numérique du clapot}

\subsection{Le paramétrage de SWAN}

Une modélisation des agitations a été réalisée avec le modèle SWAN (Simulating Waves Nearshore, Booij et al. 2004) qui permet de décrire la génération et la propagation de vagues en milieu côtier. La bathymétrie du bassin a été implantée dans une grille de calcul de mailles de $100 \mathrm{~m}$ de côté. Le code SWAN permet des calculs en modes stationnaire et non-stationnaire. En raison des variations importantes du niveau d'eau et du vent, le mode non-stationnaire, mieux adapté, a été privilégié. Le vent est actualisé toutes les $3 \mathrm{~h}$ et la hauteur de la marée (fournie par le marégraphe SHOM d'Arcachon) est introduite toutes les heures. Dans ce travail la houle océanique incidente n'est pas simulée. Les mesures ont montré qu'elle affecte le point A01 (sud de l'Ile aux Oiseaux) mais elle est inexistante dans le fond du bassin où nous testons la simulation.

Le modèle SWAN possède de nombreux réglages, ce qui nécessite de très nombreux essais avant d'aboutir à des résultats fiables, en particulier, il propose trois modèles de vent. La contribution du vent au terme source $S$ est :

$$
S(\sigma, \theta)=A+B E(\sigma, \theta)
$$

où $E$ est la densité spectrale d'énergie et $A$ et $B$ dépendent de la fréquence $(\sigma)$ et de la direction de propagation $(\theta)$ des vagues ainsi que de la vitesse et de la direction du vent. Pour le terme de croissance exponentielle $B$, SWAN propose 3 expressions dont 2 (Komen et Janssen) diffèrent dans le calcul de la vitesse de frottement. Récemment Westhuysen et al (2007) ont amélioré la dissipation par moutonnement avec une meilleure prise en compte de ce facteur limitant la croissance des vagues.

Les trois principaux mécanismes de dissipation de l'énergie des vagues sont le déferlement, le moutonnement et le frottement sur le fond. Enfin, il existe deux modèles de frottements (SWAN propose un 3e paramétrage simplifié, que nous n'avons pas utilisé avec une valeur constante de $C_{b}=0.038 \mathrm{~m}^{2} \mathrm{~s}^{-3}$ ) sur le fond dépendant de $U_{\text {rms }}$ la vitesse orbitale sur le fond :

- la méthode de Collins utilise un coefficient de frottement sur le fond donné par $C_{b}=C_{f} g U_{r m s}$, avec une valeur par défaut $C_{\mathrm{f}}=0.015$ que nous avons conservée.

- avec la méthode de Madsen, dans l'expression du coefficient de friction $C_{b}=\frac{f_{w}}{\sqrt{2}} g U_{r m s}$ le facteur $f_{\mathrm{w}}$ ne dépend que d'un paramètre de rugosité du fơnd; par défaut, il vaut $5 \mathrm{~cm}$ et d'après nos tests, nous l'avons ajusté à $2 \mathrm{~cm}$. 


\subsection{Les simulations}

Les tests ont été réalisés sur 2 dates, concernant une période de vent faible ( 6 décembre $<10 \mathrm{~m} / \mathrm{s})$ et très fort $(8$ janvier $>20 \mathrm{~m} / \mathrm{s})$. La figure 6 montre un exemple de résultat montrant l'emprise du calcul. Les résultats du modèle sont comparés aux mesures de clapot obtenues en 2 points : Le Teich et Cassy. Différents tests ont été effectués, en combinant les phénomènes physiques intégrés dans SWAN et en particulier les générations de vent (Komen, Janssen, et Westhuysen) et la prise en compte ou non de la frottement sur le fond (Madsen, Collins). Après plusieurs tests, nous avons retenu trois combinaisons : KomenCollins, Komen-Madsen et Westhuysen sans frottement. Le meilleur accord est obtenu avec la combinaison Komen/Madsen (figure 7).
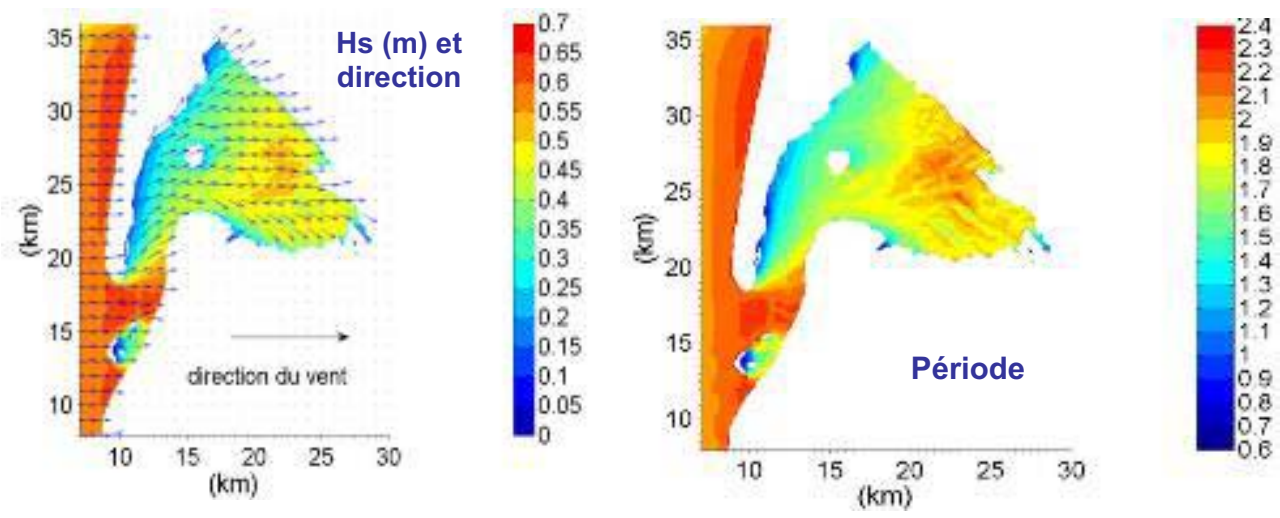

Figure 6. Exemple de simulation SWAN des agitations dans le Bassin d'Arcachon, sous l'effet d'un vent d'Ouest de $10 \mathrm{~m} / \mathrm{s}$ avec une marée de $5 \mathrm{~m}$. La houle extérieure n'est pas prise en compte afin d'évaluer le seul effet du vent.
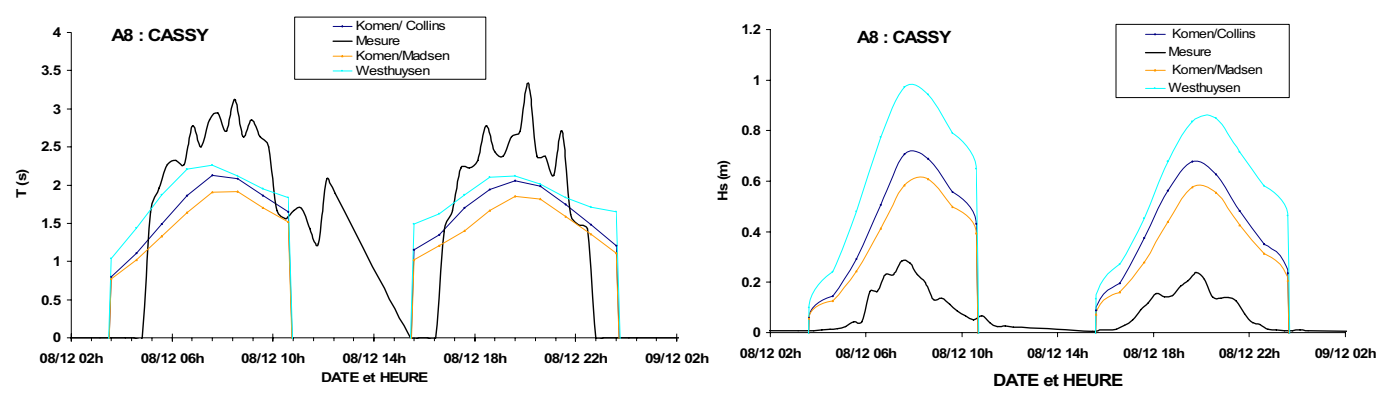

Figure 7. Comparaison entre les mesures et les simulations sur le site de Cassy (A8), lors du fort coup de vent des 7 et 8 décembre 2007. Les périodes (à gauche) sont bien reproduites alors que les hauteurs significatives (à droite) sont systématiquement surestimées. 
On peut noter une très bonne reproduction de la période du clapot de l'ordre de $2 \mathrm{~s}$ alors que les hauteurs significatives sont surestimées et avec de fortes variabilités suivant les paramètres retenus. Un facteur 3 de surestimation est énorme et on peut espérer réduire cet écart compte-tenu de l'imprécision que nous avons sur 2 paramètres importants : le régime de vent avec une valeur connue toutes les $3 \mathrm{~h}$ est trop grossier; la bathymétrie du Bassin d'Arcachon avec une résolution de l'ordre de $200 \mathrm{~m}$ n'est pas assez fine pour calculer des agitations dans le fond du Bassin où la largeur des chenaux n'excède pas $50 \mathrm{~m}$. En effet, la zone de Cassy est la plus interne de tous nos sites de mesure et c'est là où le clapot est certainement le plus délicat à calculer. D'autre part, nous ne prenons pas en compte les courants de marée qui seront couplés avec la houle dans une prochaine étude. Une autre source d'erreur pourrait être due à l'hypothèse de la pression hydrostatique pour convertir les pressions en profondeurs. Cela est certainement valide pour des profondeurs inférieures à $2 \mathrm{~m}$ mais pas au-delà, où nous sous-estimons la profondeur et donc la hauteur des vagues. Cette approximation sera abandonnée dans notre futur traitement.

\section{$5 \quad$ Conclusion}

Lors de cette étude, nous avons adapté le modèle SWAN à la bathymétrie du bassin d'Arcachon et nous avons cherché à reproduire les valeurs de la hauteur significative et de la période du clapot mesurées au dessus d'une bathymétrie complexe. Nous avons réalisé plusieurs simulations tests afin de faire le choix des paramètres et de faire une première étude des différents couplages vent/frottements. On constate que la combinaison la plus probante est le vent de Komen et la friction de Madsen, avec un coefficient de rugosité $2 \mathrm{~cm}$, et avec dissipation d'énergie. Les travaux en cours visent à mieux valider le clapot simulé, et à évaluer les contraintes de frottement générées sur le fond, qui seront intégrées dans un modèle de transport sédimentaire. D'autre part une bathymétrie plus fine et une meilleure prise en compte du vent devrait améliorer nos prévisions.

\section{$6 \quad$ Références bibliographiques}

Booij, N., Haagsma IJ.G.; Holthuijsen L.H.; Kieftenburg, A.T.M.M.; Ris, R.C.; Van der Westhuysen A.J. and Zijlema M., 2004. Swan Cycle III version 40.41. User's Manual, 115p.

Bouchet J.M., 1968. Etude océanographique des chenaux du Bassin d'Arcachon. Thèse Université Bordeaux 1, $306 \mathrm{p}$.

Christie M.C., Dyer K.R., Turner P., 2001. Observations of long and short-term variations in the bed elevation of a macro-tidal mudflat. In : Coastal and Estuarine Fine Sediment Processes; McAnally, W.H., Mehta, A.J. (Eds.). Proceedings in 
Marine Science. Elsevier Science, Amsterdam, 323-342.

Diet-Davancens J., 2006. Simulation numérique des agitations dans un domaine lagunaire : le Bassin d'Arcachon. Rapport de stage de Master 2 SUEE.

Drillon C. et Crosland E., 2007. Modélisation des agitations dans le Bassin d'Arcachon. Master I de Physique. Université Bordeaux I.

Ehrhold A., Guillou S., Auffret J.P., Garlan T., Dan Nguyen K., 2003. Bedload transport modelisation in a bay characterized by a macrotidal environment: example of the Mont-Saint-Michel Bay (France). Oceanologica Acta 26, 443-455.

Gayet J., Cuignon R., Faugères J.C., 1986. Caractères morpho-sédimentaires et processus dynamiques dans un delta édifié en milieu lagunaire mésotidal (delta de la Leyre : Bassin d'Arcachon). Bulletins IGBA n ${ }^{\circ} 39,18$ p.

Grassiat L., 1989. Hydrodynamique et évolution sédimentaire d'un système lagune-fleche littorale. Thèse doctorale, Université Bordeaux 1, $228 \mathrm{p}$.

Janssen-Stelder B., 2000. The effect of different hydrodynamic conditions on the morphodynamics of a tidal mudflat in the Dutch Wadden Sea. Continental Shelf Research 20, 1461-1479.

Le Hir P., Roberts W., Cazaillet O., Christie M., Bassoullet P., 2000. Characterization of intertidal flat hydrodynamics. Cont. Shelf Res. 20, 1433-1459.

Lecacheux, S., R. Pedreros, A. Sottolichio, E. Romieu, P. Salles, 2008. Caractérisation des vagues dans les passes du bassin d'Arcachon. Soumis aux Xèmes Journées Nationales Génie Côtier Géie Civil

Lee H.J., Jo H.R., Chu Y.S., Bahk K.S., 2004. Sediment transport on macrotidal flats in Garolim Bay, west coast of Korea: significance of wind waves and asymmetry of tidal currents. Continental Shelf Research 24, 821-832.

Quaresma V., Bastos A.C., Amos C.L., 2007. Sedimentary processes over an intertidal flat: A field investigation at Hythe flats, Southampton Water (UK). Marine Geology 241,117-136.

Pedersen J.B.T., Bartholdy J., 2006. Budgets for fine-grained sediment in the Danish Wadden Sea. Marine Geology 235, 101-117.

Perillo, G.M.E. (Ed.), 1995. Geomorphology and sedimentology of estuaries. Developments in Sedimentology, 53. Elsevier, Amsterdam, p. 471-487.

Rolinski S., Umgiesserb G., 2005. Modelling short-term dynamics of suspended particulate matter in Venice Lagoon, Italy. Estuarine, Coastal and Shelf Science 63, 561-576.

Salles, P., A. Sottolichio, P. Bretel, S. Bujan and R. Pedreros, 2008. Tidal Distortion in the Arcachon Bassin. XI International Symposium on Oceanography of the Bay of Biscay, 2-4 April, San Sebastian (Spain), Book of Abstracts "Revista de Investigación Marina", p161-p162

Verney R., 2006. Processus de contrôle de la dynamique des sédiments cohésifs. UMR 6143 M2C, These Université de Rouen. 325 p

Verney R., Deloffre J., Brun-Cotan JC., Lafite R., 2007. The effect of waveinduced turbulence on intertidal mudflats: Impact of boat traffic and wind. Continental Shelf Research, 27, 594-612.

Van der Westhuysen A., Zijlema M, Battjes J.A., 2007. Nonlinear saturationbased whitecapping dissipation. Coastal Engineering 54, 151-170 